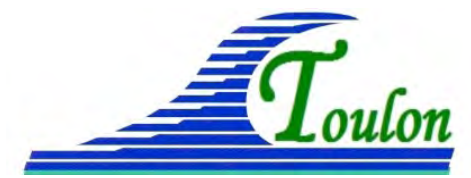
XIV $V^{\text {èmes }}$ Journées Nationales Génie Côtier - Génie Civil
Toulon, 29 juin au $1^{\text {er }}$ juillet 2016

DOI:10.5150/jngcgc.2016.068 (C) Editions Paralia CFL

disponible en ligne - http://www.paralia.fr - available online

\title{
Identification et analyse des systèmes de défense contre les submersions marines
}

\section{Céline PERHERIN ${ }^{1}$, Céline TRMAL ${ }^{2}$, Frédéric PONS $^{2}$, Céline BOURA $^{3}$}

1. Cerema (Centre d'études et d'expertise sur les risques, l'environnement, la mobilité et l'aménagement) / Direction Technique Eau, mer et fleuves, 155 rue Pierre Bouguer, BP 5, 29280 Plouzané, France.

Celine.Perherin@cerema.fr

2. Cerema (Centre d'études et d'expertise sur les risques, l'environnement, la mobilité et l’aménagement) / Direction Territoriale Méditerranée, Pôle d'activités Les Milles, 30 avenue Albert Einstein, CS 70499, 13593 Aix-enProvence CEDEX 03, France. Frederic.Pons@cerema.fr ; Celine.Trmal@cerema.fr

3. Cerema (Centre d'études et d'expertise sur les risques, l'environnement, la mobilité et l'aménagement) / Direction Territoriale Ouest, M.A.N, Rue René Viviani, 44262 Nantes, France.

Celine.Boura@cerema.fr

\section{Résumé :}

La présence d'ouvrages de protection ou d'autres obstacles naturels (topographie, cordons naturels, ...) ou anthropiques (remblais linéaires d'infrastructures) influe fortement sur l'aléa inondation. Les systèmes de défense contre les inondations sont composés de ces différents éléments structurels pour former un ensemble de défense hydraulique cohérent. Une méthodologie d'identification de ces systèmes de défense est proposée par le Cerema. Elle permet de définir le système et son fonctionnement hydraulique. Plusieurs types d'analyses peuvent être conduites suivant, d'une part, les connaissances existantes sur le territoire et, d'autre part les objectifs poursuivis.

Dans de nombreux cas, une analyse des études et données existantes est suffisante pour définir le contour des systèmes de défense (bases de données nationales et locales des ouvrages de protection, études de dangers, études d'aléa submersion marine, ...). Des analyses topographiques permettent de préciser le système de défense : confrontation de zones basses et de zones basses connectées à la mer, détection automatique d’obstacles à l'écoulement de type remblais (digues, remblai linéaires d'infrastructures, cordons naturels, etc.). D’autres analyses complémentaires, "analyses expertes" des données existantes ou la modélisation hydraulique, peuvent être utiles pour compléter l'exercice face à des systèmes de défense plus complexes.

Mots-clés : Inondation, Submersion marine, Ouvrages, Système de défense, Digues, MNT Lidar. 


\section{Thème 6 - Gestion durable des zones littorales et estuariennes}

\section{Introduction}

La gestion du risque de submersion marine s'appuie en premier lieu sur une bonne connaissance de l'aléa d'inondation. Toute action de prévention des risques (prise en compte du risque dans l'aménagement, gestion de crise, réduction de la vulnérabilité, ...) nécessite la réalisation préalable d'un diagnostic territorial quant à son exposition aux phénomènes naturels. La présence d'ouvrages de protection contre les inondations ou d'autres obstacles naturels (topographie, cordons naturels, ...) ou anthropiques (remblais linéaires d'infrastructures, ...) influe fortement sur cet aléa. Cette présence rend l'aléa variable dans le temps, en fonction de l'état de ces structures, et variable dans l'espace, en fonction de leur capacité à faire obstacle à l'écoulement et à résister en fonction de l'intensité du phénomène. Une évaluation complète et correcte de l'aléa dans des territoires "protégés", c’est-à-dire selon le sens donné ici, situés derrière des structures faisant selon certaines conditions obstacles à l'écoulement, demande donc une bonne connaissance des caractéristiques des systèmes de défense contre les submersions marines. Cette évaluation complète de l'aléa est indispensable à la gestion des inondations sur un territoire.

Plusieurs niveaux d'analyse peuvent exister suivant les objectifs poursuivis, et donc la finesse nécessaire de connaissance de l'aléa de submersion, et les connaissances existantes sur le territoire. Le présent article synthétise plusieurs types d'analyses pouvant être conduites dans un objectif d’identification des systèmes de défense, défini par son contour, et de compréhension du fonctionnement hydraulique global du système de défense.

\section{Concept du système de défense}

Les systèmes de défense contre les inondations sont composés de différents éléments structurels afin de former un ensemble de défense hydraulique cohérent assurant un certain niveau de protection d'une zone protégée. La définition conceptuelle des systèmes de défense présentée ci-dessous repose sur une analyse du fonctionnement hydraulique des sites. Elle s'inspire de l'Étude des systèmes de protection contre les submersions marines (CEREMA, 2016) et du Référentiel technique digues maritimes et fluviales (MEDDE, 2015).

\subsection{Définition générale}

Un système de défense est un ensemble d'éléments naturels ou anthropiques ayant pour effet de protéger des inondations (crues et/ou submersions marines). Il forme un contour continu et fermé définissant ainsi un milieu extérieur et une zone intérieure "protégée" où la protection est effective tant que son niveau n’est pas dépassé et les systèmes non défaillants.

Le système de défense peut être composé de structures, jouant un rôle d'obstacle à l'écoulement des eaux (digues, remblais, cordons naturels, ...), situées généralement sur 


\section{XIV èmes Journées Nationales Génie Côtier - Génie Civil \\ Toulon, 29 juin au $1^{\text {er }}$ juillet 2016}

le contour du système et d'éléments de gestion de l'eau situés dans la zone intérieure qui participent au fonctionnement du système de défense, à l'écoulement et au ressuyage des eaux (canaux, fossés, bassins de rétention, écluses, vannes, stations de pompage, etc.).

Chaque système de défense est défini autour d'une stratégie combinant un objectif de protection, au-delà duquel des entrées d'eau sont possibles dans la zone intérieure et un objectif de sûreté, au-delà duquel la tenue des ouvrages n’est plus garantie.

L'identification du système de défense est réalisée à partir d'une analyse hydraulique se basant principalement sur la topographie afin d’identifier une zone déconnectée hydrauliquement du milieu extérieur jusqu’à un certain niveau (niveau de protection).

\subsection{Représentation géographique du système de défense}

Le système de défense est défini comme un contour géographique fermé constitué par :

- des structures de défense naturelles et artificielles ;

- des lignes dites abritées représentant la limite au-delà de laquelle les territoires ne peuvent en aucun cas (même en l'absence de structures de défense) subir de submersion du fait de leurs altitudes ou de leur éloignement de la source de l'inondation.

Au sein de chaque système peuvent parfois être définis plusieurs casiers hydrauliques (voir figure 1). Ces derniers définissent au sein du système de défense des entités homogènes hydrauliquement dont le contour est constitué d’obstacles les rendant ainsi indépendants jusqu'à un certain niveau d'eau.

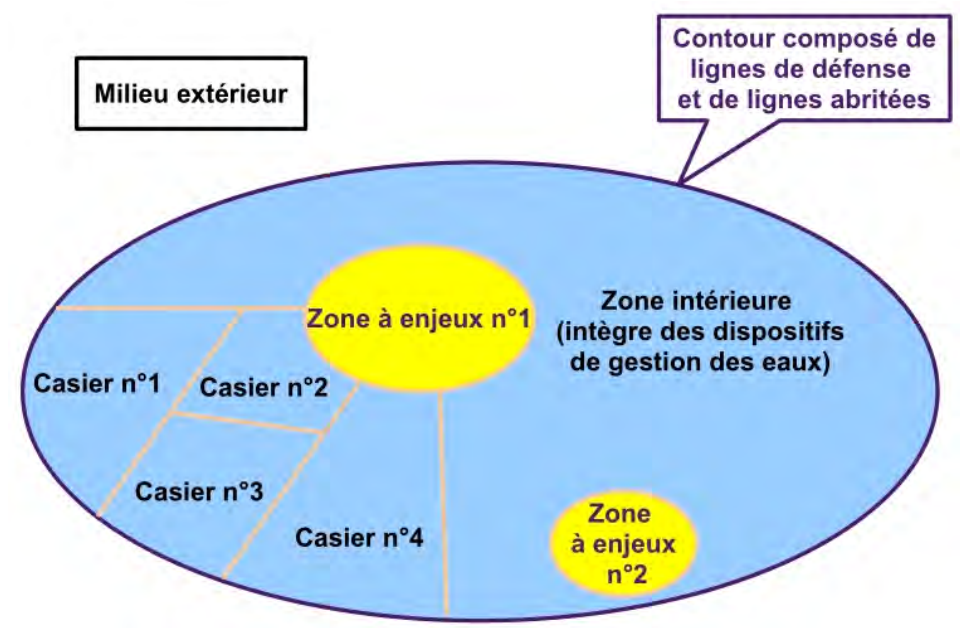

Figure 1. Représentation schématique de casiers hydrauliques au sein d'un système de défense. 


\section{Thème 6 - Gestion durable des zones littorales et estuariennes}

Ces casiers peuvent être identifiés par une analyse topographique des points hauts (ouvrages de défense, digues intérieures, remblais d'infrastructures, topographie naturelle, etc.) complétée par une analyse des échanges hydrauliques entre les secteurs bas séparés par ces points hauts. Ces casiers de diverses tailles ont chacun des vitesses de montée des eaux différentes liées à leurs caractéristiques.

A partir de ce concept, une méthodologie d'identification et d'analyse de ces systèmes de défense est proposée. Elle permet de définir le contour du système, et ainsi de préciser la zone "protégée" intérieure, et le fonctionnement hydraulique global du système de défense qui a pour objectif de limiter les conséquences négatives des inondations sur les enjeux de cette zone. La définition et l'identification des systèmes de défense proposées ici reposent donc sur une analyse du fonctionnement hydraulique des sites, permettant ainsi d'identifier les systèmes de défense intégrant des structures aménagées ou non dans un objectif de protection des inondations et dont le rôle de protection contre les inondations n’aurait pas nécessairement encore été identifié.

\section{Analyse des connaissances existantes}

\subsection{Les connaissances sur les structures de défense}

Les recensements existants des structures de défense contre les inondations, principalement des digues, sont à analyser en premier lieu. Ils peuvent donner lieu à l'alimentation de bases de données nationales comme SIOUH ou locales tel que DIGSURE soutenu par l'association France Digues. Les structures faisant obstacle à l'écoulement (cordons naturels, remblais) mais également des éléments de gestion des eaux (canaux, étiers, clapets, écluses, pompes, etc.) doivent être recensés. La cote de crête des structures de défense est importante à identifier.

Les études de dangers, réalisées par le responsable de l'ouvrage, permettent d'obtenir une définition des systèmes de défense et des caractéristiques des ouvrages. Cette source d'information est à privilégier dès qu'elle est disponible. Les études de dangers définissent, selon leur qualité, tout ou partie des éléments suivants : le système de défense, la zone intérieure "protégée", le niveau de protection, les sollicitations auxquelles sont soumises les structures et leurs défaillances possibles, les conséquences possibles sur la zone intérieure en cas de dépassement du niveau de protection. Les études de dangers contiendront ainsi l'ensemble des informations nécessaires à la réalisation de cette première étape de définition des systèmes de défense.

\subsection{Les études hydrauliques et les données de description physique du littoral}

En l'absence d'études de dangers, les études hydrauliques existantes permettent d'apporter des informations complémentaires utiles à la caractérisation des systèmes de défense et à la compréhension de leur fonctionnement hydraulique. Ces études peuvent être des Atlas de Zones Inondables (AZI), des études d'aléas d'inondation préalables à 


\section{XIV èmes Journées Nationales Génie Côtier - Génie Civil \\ Toulon, 29 juin au $1^{\text {er }}$ juillet 2016}

la réalisation de Plans de Prévention des Risques d'Inondation (PPRI), les cartographies issues de la Directive "Inondations" ou encore des études préalables à la réalisation d'un aménagement de protection.

L'ensemble de ces études hydrauliques peut être exploité pour définir le système de défense, et notamment :

- identifier les structures de défense jouant un rôle de protection,

- identifier les éléments de gestion des eaux au sein du système, et les limites de la (ou des) zone(s) impactée(s) par l'effet de ces éléments,

- définir l'emprise de la ou des zone(s) inondable(s), donnant une première approche de la zone intérieure "protégée",

- définir un niveau de protection des structures de défense ou à défaut un niveau de protection apparent par la connaissance des cotes de crêtes de ces structures,

- définir les casiers hydrauliques du système de défense du fait de l'analyse des écoulements au sein de la zone "protégée".

En l'absence d'études hydrauliques, une connaissance générale du site par la confrontation de données cartographiques de connaissance physique du littoral (données topographiques, entités hydrographiques, infrastructures structurantes) et/ou de photographies aériennes, facilement mobilisables dans un SIG, est utile à la définition des systèmes de défense et de leur zone "protégée". Cette définition passe par l'intermédiaire d'une analyse experte de la topographie et des obstacles à l'écoulement.

\section{Analyses topographiques}

Les analyses topographiques développées dans ce cadre peuvent aider à la compréhension en l'absence d'études locales spécifiques. Elles peuvent être menées partout où sont disponibles des modèles numériques de terrain de haute résolution de type Lidar. Ces analyses topographiques permettent d'une part, de détecter les remblais qui peuvent faire barrière à la submersion et participer au système de défense et, d'autre part, d’approcher le contour de la zone intérieure "protégée".

\subsection{Détection automatique des remblais}

Un des points clés de la définition des systèmes de défense est l'identification des digues, remblais linéaires d'infrastructures, cordons naturels, etc. qui peuvent faire obstacles à la submersion. Ces formes peuvent être identifiées sur des modèles numériques de terrain avec des outils automatiques permettant si besoin de traiter de grands espaces en peu de temps.

L’algorithme développé (TRMAL et al., 2016) s’appuie sur le calcul de dénivelé autour d'un point. Il traduit mathématiquement un principe simple : la détection d'une forme linéaire dont la cote se situe plus haut que celle du terrain naturel de part et d'autre. Pour approcher ce constat simple, le calcul se fait sur une certaine largeur de part et d'autre d'un point, cette largeur est réglable et permet de détecter des structures plus ou moins 


\section{Thème 6 - Gestion durable des zones littorales et estuariennes}

larges. Le résultat est un fichier raster contenant les dénivelés calculés (différence entre la cote d'un point haut et de deux points situés de part et d'autre). En comparant les résultats donnés pour plusieurs largeurs de calcul (distance entre le point haut et les points adjacents), les largeurs des formes sont estimées (voir figure 2).

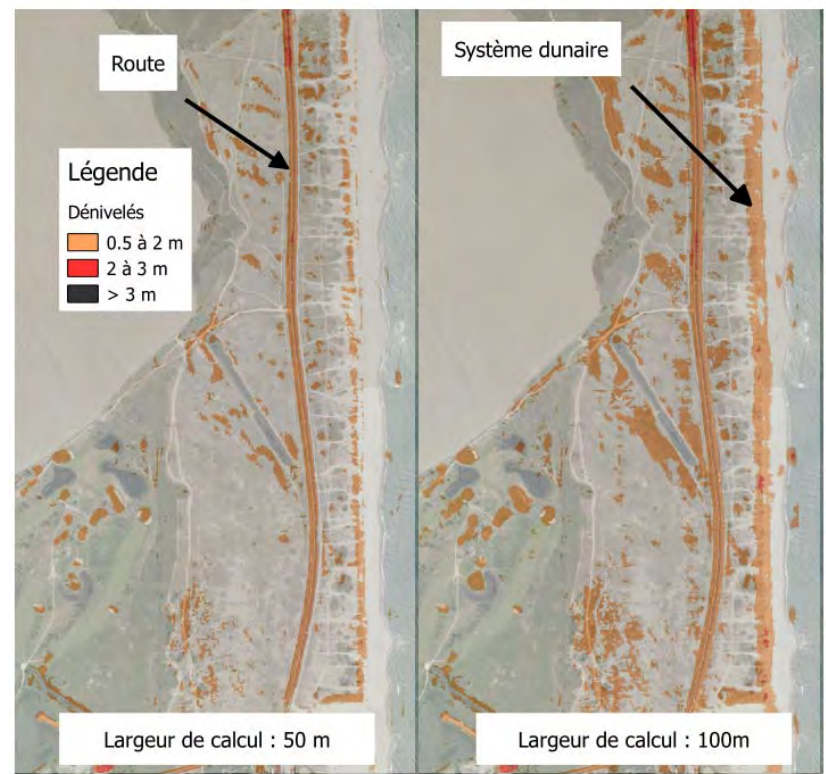

Figure 2. Détection de structures de type dunaire (entre Saint-Cyprien et Canet, 66).

\subsection{Identification des zones basses et des zones connectées à la mer}

La confrontation de deux procédés d'analyse topographique des zones basses littorales permettent d'améliorer la connaissance des zones "protégées". Ces deux procédés s'appuient sur des outils SIG disponibles dans le logiciel Qgis/Grass.

La première analyse topographique est classique. Les zones inférieures à une certaine altitude, correspondant à un niveau marin donné, sont identifiées, qu'elles soient ou non éloignées de la mer. La deuxième analyse topographique utilisée permet d'identifier uniquement les zones basses connectées à la mer (TRMAL \& PONS, 2015).

Pour un niveau marin donné, la différence d'extension entre la première analyse et la deuxième permet de faire ressortir les zones basses non connectées à la mer pour ce niveau et donc potentiellement protégées pour ce niveau.

Appliquer cette méthode à différents niveaux marins espacés d'un pas constant (par exemple $25 \mathrm{~cm}$ ) permet d'assimiler, en première approche, le niveau de protection apparent au niveau marin maximum qui n’inonde pas le casier (voir figure 3).

Ce procédé permet également, pour chaque casier, d'approcher la cote des structures de défense, du moins d'identifier leur point bas, avant le début d'inondation du casier.

La qualité du résultat dépend en grande partie de la qualité du MNT utilisé car l'altitude des pixels est analysée de proche en proche pour "diffuser" l'inondation. Les limites de 


\section{XIV èmes Journées Nationales Génie Côtier - Génie Civil \\ Toulon, 29 juin au $1^{\text {er }}$ juillet 2016}

la méthode sont également liées à l'algorithme qui inonde de pixel en pixel avec une "diffusion" pouvant se faire en diagonal, ce qui signifie que par un seul point bas d'un mètre de large, un volume infini inonde la zone basse en arrière. La méthode a donc tendance à affecter un niveau de protection apparent de la zone inférieur à la réalité.

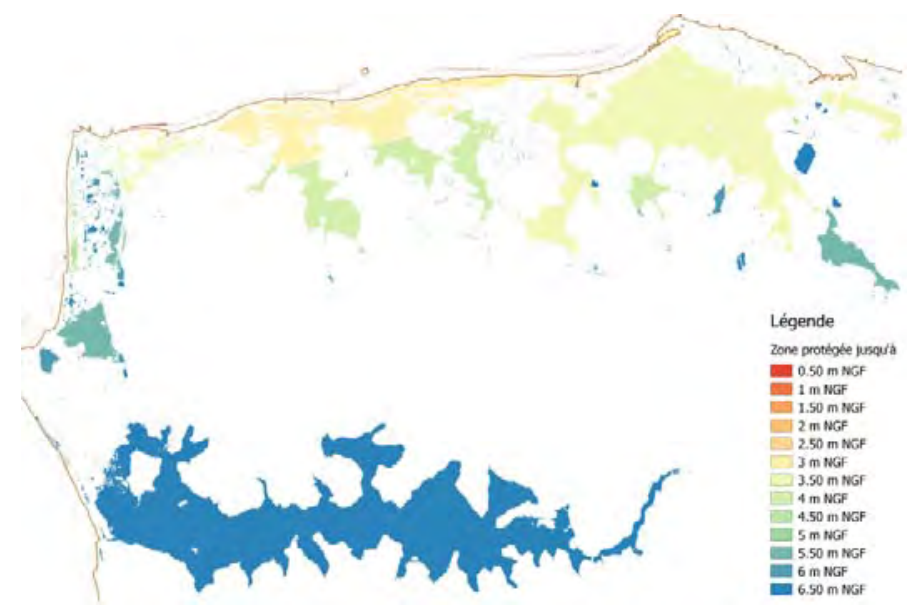

Figure 3. Identification de la zone "protégée", de ses casiers, et leur niveau de protection apparent (site de Corsept et Saint-Brevin-les-Pins, 44).

\section{Analyses complémentaires}

5.1 Estimation des zones submergées et du contour du système de défense

Les méthodes d'analyses topographiques précédentes sont utiles à une première estimation des zones potentiellement "protégées" et des hauteurs d'eau potentielles au sein du système. Les limites des zones potentiellement "protégées" permettent d'estimer la limite qui ferme le contour du système en complément des structures de défense. Des analyses expertes complémentaires peuvent être utiles pour estimer la pertinence de ces méthodes au regard des caractéristiques de la zone.

La projection d'une cote donnée sur la topographie pour définir les zones soumises à un aléa d'inondation présente des limites, notamment à distance des ouvrages et lorsque la surface de la zone "protégée" est importante, du fait de la dynamique de propagation de l'onde de submersion sur de grandes étendues. Pour estimer cette limite, une analyse des volumes d'eau peut être réalisée en comparant le volume apporté (défini à partir d'un marégramme) au volume inondé par la projection. Cette différence de volume et une analyse fine de la topographie, identifiant les obstacles à l'écoulement (reliefs ou structures de défense), permettent d'estimer plus finement l'emprise des zones inondées pour un tel niveau. Lorsque la topographie est complexe, une modélisation hydraulique est utile à l'identification des obstacles successifs, et ainsi des casiers hydrauliques, à partir des cheminements successifs de l'onde de submersion. Elle permet aussi d’approcher plus finement les limites des zones submergées. 


\subsection{Analyse de fréquences d'inondation complémentaires}

Les analyses ci-dessus sont effectuées pour un niveau marin donné correspondant au niveau de protection apparent sans tenir compte de défaillances du système de défense. Afin de correctement appréhender les conséquences d'une inondation, le choix des niveaux marins testés (et donc des fréquences d'événements) doit, dans la mesure du possible, être en lien avec la connaissance du comportement des ouvrages : niveau de surverse, niveau engendrant des dommages structurels, ... Plusieurs scénarios d'inondation, choisi en fonction des caractéristiques du système de défense et des structures le composant, sont donc à étudier pour une analyse détaillée du fonctionnement hydraulique du système. De telles études sont menées dans le cadre des études préalables aux Analyses Coûts-Bénéfices (ACB) afin de définir les courbes fréquences-dommages. La modélisation numérique peut alors raffiner la connaissance du fonctionnement hydraulique d'un système complexe, comportant plusieurs casiers.

\section{Conclusions}

Ces méthodes sont appliquées dans l'objectif d'identifier les éléments de défense jouant un rôle de protection contre les inondations et le système complet de défense. Le choix des méthodes doit être adapté aux connaissances existantes et données disponibles mais également aux caractéristiques du territoire à investiguer (étendue, relief, etc.).

Ces analyses seront utiles notamment dans le cadre des actions relevant de la compétence GEMAPI, afin d'adapter les mesures de gestion de ces éléments de défense en lien avec les enjeux de la zone protégée.

\section{Références bibliographiques}

CEREMA -Centre d'Études et d'Expertise sur les Risques, l'Environnement, la Mobilité et l'Aménagement- (2016). Étude des systèmes de protection contre les submersions marines - Méthodologie et études de cas issues du retour d'expérience Xynthia. Rapports DtecEMF, à paraître.

MEDDE -Ministère de l'Ecologie, du Développement Durable et de l'Energie- (2015). Référentiel technique digues maritimes et fluviales. Groupe de Travail "Référentiel technique digues maritimes et fluviales", janvier 2015, 191 p. Disponible en ligne: http://www.eau-mer-fleuves.cerema.fr/le-referentiel-technique-digues-maritimes-et-a1418.html

TRMAL C., PONS F. (2015). Manipulation des MNT haute résolution pour détecter les obstacles aux submersions marines et cartographier les phénomènes - Exemples sur la côte méditerranéenne française. $3^{\text {ème }}$ Conférence Méditerranéenne Côtière et Maritime, Ferrarra, Italie, pp 231-234. http://dx.doi.org/10.5150/cmcm.2015.045

TRMAL C., PONS F., LEDOUX (2016). Flood protection structure detection with Lidar: examples on French Mediterranean rivers and coastal areas. FLOODrisk 2016, à paraître. 